\title{
Vector Feedback Homogeneity and Inner Layout Influenc on Fluxgate Sensor Parameters
}

\author{
Vojtěch Petrucha, Michal Janošek, and Marco A. Azpúrua
}

\begin{abstract}
Vector feedback is a concept which can significantly improve linearity and stability of a magnetic field sensor. The feedback coils effectively cancel the measured magnetic field in the inner volume of the triaxial sensor. Thus, in case of fluxgates, it suppresses one possible source of nonlinearitycross-field sensitivity error. The triaxial sensor axes orthogonality should be primarily defined by the orientation of the feedback coils, while the sensitivities are defined by feedback coil constants. The influence of the homogeneity of the feedback field and the influence of the sensor inner layout on calibration parameters of a vectorially compensated triaxial fluxgate magnetometer are presented.
\end{abstract}

Index Terms-Field homogeneity, fluxgate sensor, scalar calibration.

\section{INTRODUCTION}

$\mathbf{T}$ HERE is a long-term effort to develop high-precise fuxgate magnetometers at the Department of Measurement, Faculty of Electrical Engineering, Czech Technical University in Prague. A typical application is in vertical and horizontal underground drilling navigation, archeological and geological prospection, or Earth's magnetic f eld observation for scientif c purposes. Fluxgates sensors offer the lowest noise densities of all vector magnetic sensors working at room temperatures $(<3 \mathrm{pT} / \sqrt{ } \mathrm{Hz}$ at $1 \mathrm{~Hz})$ while having very high dynamic range $(>120 \mathrm{~dB})$ and excellent time - temperature stability [1]. One of the latest development steps at our department is an application of vector compensation. The vector compensation of measured magnetic fiel brings several benefits First, it almost completely eliminates the cross-fiel effect (sensitivity to perpendicular magnetic feld). Second, the sensitivity and orthogonality should be define primarily by the robust and mechanically stable compensation coils system [2]. The vector compensation design brings complexity and increases price, but the benef ts are more important when the high precision is a goal.

The feedback coils should ideally provide homogenous compensation feld in the inner volume of the triaxial sensor

Manuscript received June 21, 2014; revised September 5, 2014; accepted September 24, 2014. This work was supported by the Czech Science Foundation under Grant 13-39088P. The Associate Editor coordinating the review process was Dr. Theodore Laopoulos.

V. Petrucha and M. Janošek are with the Department of Measurement, Faculty of Electrical Engineering, Czech Technical University in Prague, Prague 166 36, Czech Republic (e-mail: petruvoj@fel.cvut.cz).

M. A. Azpúrua is with the Department of Electronic Engineering, Universitat Politècnica de Caralunya, Barcelona 08034, Spain (e-mail: marco.azpurua.a@ieee.org).

Color versions of one or more of the f gures in this paper are available online at http://ieeexplore.ieee.org.

Digital Object Identifie 10.1109/TIM.2014.2362831 body where the magnetic cores of the individual fuxgate sensors are present. To further improve the properties of a compact vectorially compensated fuxgate sensor [3], we enhanced the homogeneity of the feedback coils and studied its effect on calibration parameters of the triaxial sensor. Calibration parameters (three sensitivities, three orthogonality angles, and three offsets) are evaluated by scalar calibration technique [4]. The technique provides accurate and well repeatable results and requires only basic equipment. The impulse to conduct this research were nonideal values of orthogonalities we got while calibrating the former sensor [3].

In addition to [5], we evaluated also the inf uence of the inner layout of the individual sensing elements. Former design uses three separate single-axis fuxgate sensors that are embedded into the vector compensation system structure. This design is convenient because it allows to completely test and tune each single axis fuxgate sensor for main parameters (noise and offset) before the whole triaxial sensor is fully assembled. However, this layout places the individual sensor magnetic cores asymmetrically with respect to the center of symmetry of the feedback coil system. Based on the results of the feedback f eld homogeneity inf uence, we proposed another inner layout, partially similar to the design presented in [6], which uses only two magnetic cores. Two ring-shaped f uxgate cores are placed symmetrically in the geometrical center of the feedback system. New triaxial sensor that implements this modif ed dual-core inner design was built and evaluated.

The main goal of the research was to improve the orthogonality of the vectorially compensated triaxial fuxgate sensor. However, there was an assumption that the compensation f eld homogeneity and inner structure topology could inf uence also the behavior of offset temperature dependences, as there are microscopic movements or rotations of the individual $\mathrm{f}$ uxgates sensors in the vector compensation body when the temperature is changed. This could theoretically mean that the offset will be less affected in a compensation system with better homogeneity or in a more symmetric topology of the inner sensing elements. Experiments to study this behavior were conducted.

\section{SENSOR DESCRIPTION}

The vectorially compensated triaxial vector f uxgate sensor consists of several parts. In the middle, there are usually three single-axis fuxgate sensors. Each of them has a magnetic core, and excitation and pickup windings. The fuxgate principle is well known, the soft magnetic core is periodically deeply saturated by the current fowing through the excitation wind- 


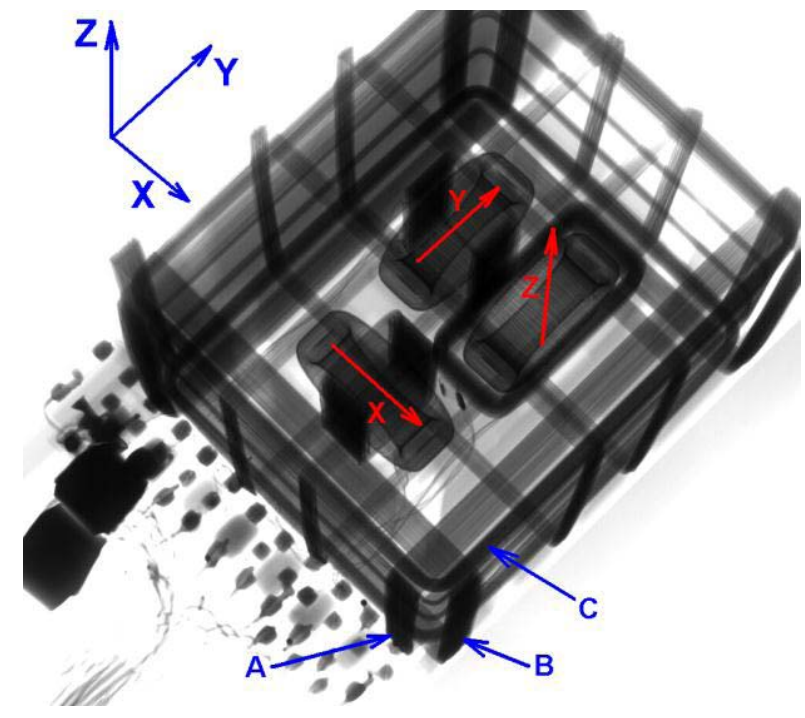

Fig. 1. X-ray image of the former sensor design (02). Each set of feedback coils has four sections. A-C mark the sections where the extra turns were added ( $\mathrm{A}$ is a part of $x$-axis compensation, $\mathrm{B}$ is a part of $y$-axis compensation, and $\mathrm{C}$ is a part of $z$-axis compensation).

ing. Polarity and amplitude of the measured magnetic fiel is related to the phase and amplitude of the second-harmonic voltage sensed across the pickup winding. The single-axis sensor works as a zero detector and its linearity is significa tly improved when compensation principle is used. Feedback current is then fed directly to the pickup winding or separate feedback coil is used. In case of the vectorially compensated sensor, all the three components of measured magnetic feld vector are compensated simultaneously in the inner volume of the triaxial sensor [2].

Four different sensor designs were used to evaluate the homogeneity and inner structure effects. The sensors are later referenced in the text as $(02,03 a, 03 b, 04)$. Sensor marked as (02) is the oldest design. It consists of three single-axis ring-core fuxgate sensors embedded in a compensation structure with external dimensions of $47 \mathrm{~mm} \times$ $40 \mathrm{~mm} \times 40 \mathrm{~mm}$. The effective core diameter is approximately $13 \mathrm{~mm}$ and total dimensions of a one single-axis sensor are $20 \mathrm{~mm} \times 20 \mathrm{~mm} \times 10 \mathrm{~mm}$. The magnetic core material (amorphous ribbon, $2.5 \mathrm{~mm}$ width) is Vitrokov 8116 wound on a toroidal support made of BNP-2 machinable ceramics. The feedback coils are of slightly modifie Merritt [7] four square-coil arrangement for each axis. Fig. 1 presents the X-ray image of this former design.

With the help of semianalytical technique [8], we were able to further improve the feedback feld homogeneity in all three axes, which was verifie by Finite Element Method (FEM) modeling (ANSYS Magnetostatic). The $x$ - and $z$-axes were redesigned to three coils, while only the coil spacing and turns count were modif ed for $y$-axis. The details concerning the homogeneity improvement are mentioned in Section III. This new feedback system design was used for sensors (03a) and (03b). There are also some other modif cations with respect to sensor (02). The magnetic cores of the individual single-axis sensors were made of a specificall magnetic fiel annealed Metglas 2714 amorphous ribbon. The Metglas 2714 provides

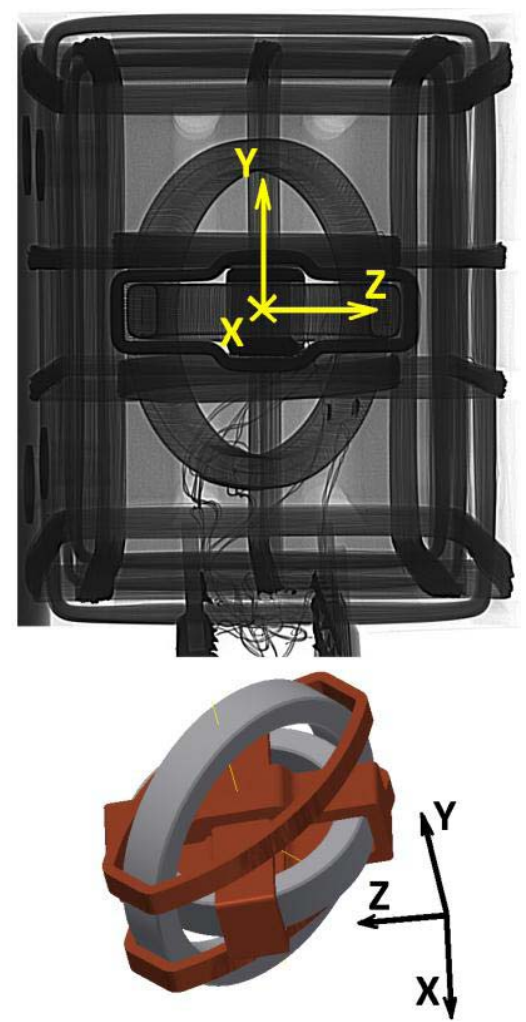

Fig. 2. Side X-ray view of the sensor (04) with the new dual-ring-core inner structure (top). Model of the dual-ring-core design shows the placement of all pickup coils (bottom). The $y$-axis uses ring core with a slightly higher diameter, which is placed in a diagonal plane with respect to $x-$ and $z$-axes. The pickup coil is splitted into two parts in case of $y$-axis.

lower noise at lower excitation energy, which helps to prevent self-heating of the sensor head. Sensor (03a) uses the same design of the three ring-core single-axis sensors as sensor (02). To evaluate properties of another design, the triaxial sensor (03b) was built using miniature race-track cores. The racetrack cores were designed to $\mathrm{ft}$ exactly into the same space as the former ring-core fuxgates, but due to its shape, the length of the race-track is higher than the diameter of the ring. We expected to gain lower noise, but actually the design provided only a small improvement but the construction was very demanding and expensive.

Sensor (04) has a completely different inner structure. It consists of two ring cores with a slightly different diameter, which are embedded one in another in the center of symmetry of the whole sensor. All pickup coils were made as selfsupporting coils enabling faster and easy assembly of the whole structure (Fig. 2). The two ring cores were designed to have signif cantly higher effective diameter of the magnetic cores (22 and $28.7 \mathrm{~mm}$ ), which considerably helps to decrease sensor noise. The smaller ring core has two mutually perpendicular pickup coils, one for $x$-axis and second for $z$-axis. The bigger ring core is placed perpendicularly with respect to the smaller ring and carries the $y$-axis pickup coil which is split symmetrically into two parts. The bigger ring is tilted with respect to $z$-axis by $45^{\circ}$ to better $\mathrm{ft}$ into the space available in the compensation support.

The feedback coils support was made of PEEK GF30 engineering plastic in case of sensor (02), which was changed 
TABLE I

Summary of THE SEnSORs Used For the MEASUREMENTS

\begin{tabular}{c|c|c|c} 
Sensor & Inner topology & Feedback design & Material \\
\hline (02) & $3 \times$ ring-core & Modified Merritt & PEEK GF30 \\
\hline (03a) & $3 \times$ ring-core & Semi-analytical & MACOR \\
\hline (03b) & $3 \times$ race-track & Semi-analytical & MACOR \\
\hline (04) & $2 \times$ ring-core & Semi-analytical & PA3200GF
\end{tabular}

for sensors (03a) and (03b) to MACOR. The machinable ceramics is mechanically more stable with respect to temperature changes but is also unfortunately much more brittle. The support is made of two halves; each of them has milled grooves for the coils and a cavity for the set of three singleaxis fuxgates or the new dual-core structure. There is also an excitation resonant capacitor visible in Fig. 1 as well as the connecting terminals. The compensation support was made by 3-D printing in case of sensor (04) to speed up the manufacturing process and to easily produce relatively complicated part that supports the pickup coils. The 3-D printer used glass-f lled polyamide PA3200GF. See Table I for summary of the used sensors.

\section{Enhacement of Field Homogeneity}

Scalar calibration technique [4] is used during the sensor development to evaluate sensor properties. The method provides very accurate and well repeatable calibration information: three sensitivities, three orthogonality angles, and three offsets. By orthogonality, we understand the alignment between an ideal orthogonal reference frame of the triaxial sensor and the frame made of real magnetic sensitivity axes that come out from the scalar calibration algorithm. The magnetic axes should be aligned with the compensation coils mechanical axes in the case of a vectorially compensated sensor. The algorithm is based on mathematical processing of data-samples that are collected with the calibrated instrument in a homogenous magnetic feld. The samples must cover the whole measurement range of the triaxial sensor for all the three sensitivity axes to get reliable results.

Nevertheless, the orthogonality was worse $-0.44^{\circ}$ for sensor (02) than we have expected considering the precision of machining and assembly $\left(<0.1^{\circ}\right)$. There was an assumption that the higher orthogonality error comes from poor homogeneity of the compensation feld or the nonideal nonsymmetrical placement of the three single-axis f uxgate sensors in the inner volume of the compensation coils. We preferred to keep the former topology of placement of the three single-axis sensors because it brings some benefit with respect to more complicated but more symmetric layouts [6]. Even the stateof-the-art sensors use similar approach [9]. Each single-axis sensor can be individually tuned for minimum feedthrough signal and noise before the assembly. Therefore, at frst, we considered the enhancement of the homogeneity of the compensation f eld as an option.

The temperature dependence of the orthogonality has been measured using a nonmagnetic thermostat and multiple scalar calibrations at different temperatures [10].
TABLE II

FIELD HOMOGENEITY IMPROVEMENT

\begin{tabular}{c|c|c|c} 
Field homogeneity $\boldsymbol{H}$ & \multicolumn{3}{|c}{ Axis } \\
\hline & $\mathrm{X}$ & $\mathrm{Y}$ & $\mathrm{Z}$ \\
\hline Former "Merritt" & $92.98 \%$ & $97.4 \%$ & $91.57 \%$ \\
\hline New "Semi-analytical" & $97.62 \%$ & $99.1 \%$ & $96.46 \%$
\end{tabular}

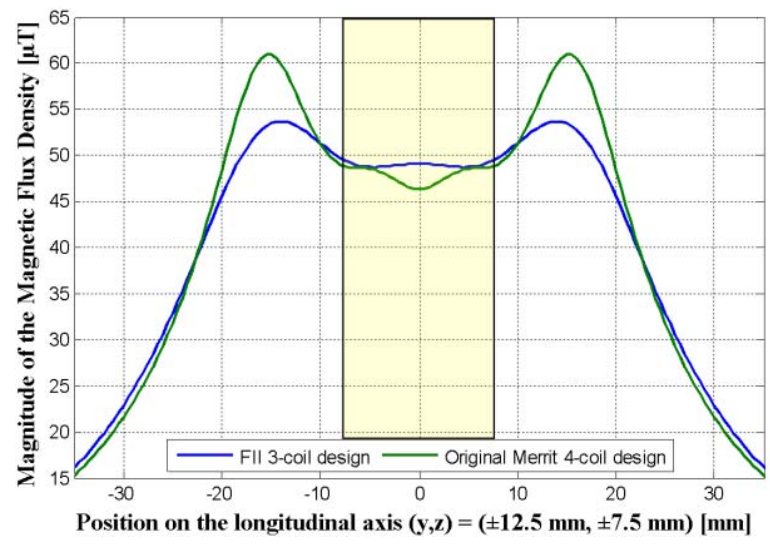

Fig. 3. Magnetic flu density distribution for $x$-axis; comparison of former and new design, region of interest is marked in the middle of the picture (simulation output, using calculation from Biot-Savart law [8]).

Relatively strong dependence of the orthogonality has been found (up to $0.38 \operatorname{arcsec} /{ }^{\circ} \mathrm{C}$ ) and thus it is very welcomed to decrease the nonorthogonality and thus possibly decrease its temperature variations.

The former feedback coils design is based on Merritt fourcoil setup slightly modifie with the use of FEM modeling to make the support machinable. Nevertheless, it was diff cult to evaluate the magnetic fiel homogeneity in the volume of the sensors. The FEM modeling software usually allows evaluating the magnetic fux density along a path or a plane but not the homogeneity in a specif $\mathrm{c}$ volume. To overcome this problem, the semianalytical method has been used [8]. This method uses analytical calculation of the magnetic fiel from Biot-Savart law and iterative search algorithm that uses objective function (average magnitude of the magnetic fux density in the volume of interest) to fnd best values for coil section spacing and number of turns. The algorithm uses some simplificati ns that make the processing faster-coils are considered ideally thin and it does not support any ferromagnetic cores. The method provided signif cant improvement over the initial design, as is summarized in Table II. The homogeneity $H$ is evaluated with respect to (1), $B_{\mathrm{MAX}}$, and $B_{\mathrm{MIN}}$ being the maximal and minimal values, and $B_{\mathrm{MEAN}}$ the mean value of magnetic $\mathrm{fl} \mathrm{x}$ density in the volume of interest. The results were later conf rmed by FEM simulation in ANSYS Magnetostatic and Flux3-D. The $x-$ and $z$-axes were redesigned to have only three sections, which is also favorable for machining and coil winding. The new design of $y$-axis has still four sections, but the spacing and number of turns were slightly modif ed. Fig. 3 shows the plot of magnetic fl $\mathrm{x}$ density in $x$-axis for original four-coil modif ed Merritt 
design and new three-coil semianalytical design. In the middle of the picture is marked a region of interest, where the fuxgate sensor cores are positioned. The change in coils spacing led to minor increase of the compensation system dimensions $(50 \mathrm{~mm} \times 40 \mathrm{~mm} \times 40 \mathrm{~mm})$

$$
H=\left(1-\frac{B_{\mathrm{MAX}}-B_{\mathrm{MIN}}}{B_{\mathrm{MEAN}}}\right) 100 \% .
$$

\section{Two-RIng-CORE INNER Structure}

To evaluate the possible inf uence of inner parts symmetry on triaxial sensor parameters, we proposed new design of the fuxgate sensing elements. Our experience indicates that fuxgate ring-core sensors with higher effective diameter of the magnetic core provide lower noise [11]. Therefore, we extended the inner volume of the feedback support to maximize the possible ring-core diameter. The external dimensions of the compensation support remained the same $(50 \mathrm{~mm} \times 40 \mathrm{~mm} \times 40 \mathrm{~mm})$. The dimensions of a cavity that accommodates the sensing elements are $36 \mathrm{~mm} \times 28 \mathrm{~mm} \times 28 \mathrm{~mm}$. There are only two magnetic cores. The smaller ring (external diameter of $24 \mathrm{~mm}$ ) is shared by $x$ - and $z$-axis pickup coils. The bigger ring (external diameter of $30.7 \mathrm{~mm}$ ) is placed in a diagonal plane with respect to $x$ - and $z$-axis to $\mathrm{ft}$ into the cuboidal cavity in the feedback coils support. This design (04) is perfectly symmetrical, but because of its increased size, the magnetic cores experience even higher $\mathrm{f}$ eld nonhomogeneity than in the previous designs (02) and (03). For simplicity, we used the same improved homogeneity feedback coil design as was developed for sensor (03). This actually means that the results will provide stronger comparison between the feld homogeneity versus placement symmetry effect.

\section{EXPERIMENTS}

\section{A. Field Homogeneity Influenc on Orthogonality}

The real infuence of the feedback magnetic feld nonhomogeneity on orthogonality angles was investigated. The feedback f eld nonhomogeneity has been created by changing the symmetry in turns count in the modif ed Merritt four-coil design by adding or subtracting turns from one outer coil. The FEM simulation conf rms that a gradient of approximately $\pm 66 \mu \mathrm{T} / \mathrm{m}$ is created (Fig. 4).

To decrease the f eld homogeneity, twelve extra turns were added to each one outer section of the $x, y$, and $z$ feedback coils (Fig. 1), together with switches, which allowed to disconnect the turns or to connect them in series or antiseries with the basic turns. We evaluated the inf uence by conducting a number of scalar calibrations [4] for various extra-turns conf gurations (extra turns active for $x, y, z, x y, x z$, and $y z$ axes, tested in both polarities). The $x, y$, and $z$ outer sections have nominally 186,196 , and 189 turns, respectively, and in total 492, 558, and 462 turns.

At frst, we evaluated the inf uence of extra turns active only in one axis, and later we also tried some other possible combinations of extra-turns active in two axes simultaneously. Together we evaluated 15 combinations: $000,+00,-00$, $0+0,0-0,00+, 00-,++0+-0,-+0,-0,+0+,-0-$,

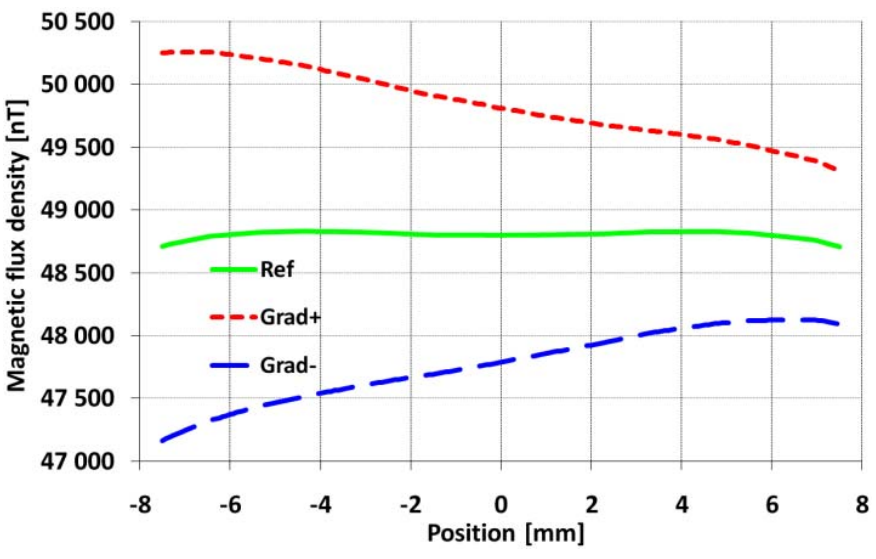

Fig. 4. Simulation of feedback fiel nonhomogeneity created in $x$-axis by connecting the extra turns in series $(\mathrm{Grad}+)$, anti series $(\mathrm{Grad}-)$, and the normal f eld (Ref). The nonhomogeneity is $\sim \pm 66 \mu \mathrm{T} / \mathrm{m}$. Sensor center is in $0 \mathrm{~mm}$ position.

$0++$, and $0-(0$ means extra turns not connected, + extra turns connected in series, and - extra turns connected in antiseries, applies for compensation coils $x, y$, and $z$ ). For each combination, at least two scalar calibrations were done to be sure that the results are repeatable.

\section{B. Influenc of the Inner Layout of the Individual Sensing Elements}

The sensor (04) with modif ed inner topology has been built and calibrated using the scalar calibration technique [4]. We can estimate the inner layout inf uence by comparing the calibration results for different inner topologies (03a) and (03b) versus (04). We repeated the same approach with introduced feedback f eld nonhomogeneity by adding the extra turns also to triaxial sensor (04). We supposed that a comparison of the results of scalar calibrations for different introduced feedback fiel nonhomogeneities can provide useful information about the inf uence of the inner layout topology.

\section{Compensation Field Homogeneity Influenc on Offset Temperature Dependence}

Theoretically, feedback fiel gradient along the respective sensor should also affect the offset temperature dependence because of the sensor changing its position due to thermal expansion. Another experiment was performed to test this influence The sensor was placed in a six-layer permalloy magnetic shielding and was equipped with a thermostated box that allowed control over the sensor temperature. Computerbased data-logging system has been assembled, logging the sensor temperature and the three magnetometers' outputs. The switching of feedback extra turns was also manual in this case. The measurement setup is presented in Fig. 5. The magnetic shield is insulated from the thermostated chamber by a Dewar f ask. The bif larly wound heat exchanger placed in the Dewar is cooled/heated by a liquid circulated with a gear pump in a closed loop from an external thermostat. The usage of a Dewar fask ensures that the temperature of the inner layers of the permalloy shielding stays constant and thus there are 


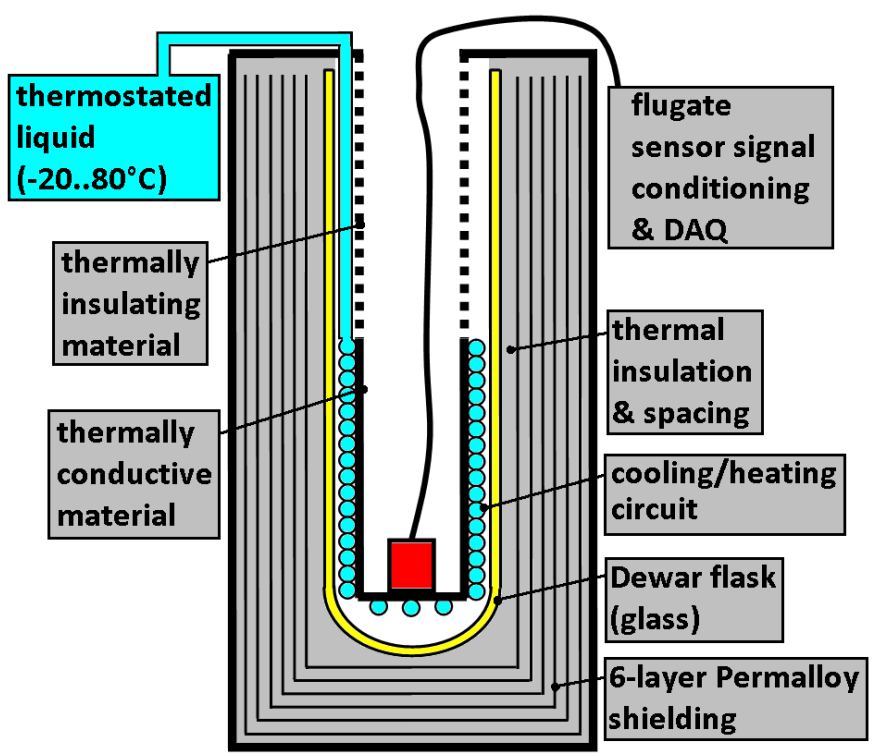

Fig. 5. System for evaluation of offset temperature dependence of the fluxgat sensor.

no thermally induced variations in the residual magnetic fiel of the shielding.

\section{RESULTS}

\section{A. Evaluation of the Field Homogeneity Influenc on Orthogonality}

During two measurement campaigns, over 38 scalar calibrations were made with various data length (56 positions or 161 positions), each extra-turn combination was measured two or three times. The sensitivities were affected as was expected from the simulation - the extra turns changed the coil constant and thus the sensitivity in appropriate axis, which was compensated in the later calculations.

On the other hand, the behavior of orthogonality angles is more complex. The scalar calibration [4] uses three angles that defin the orthogonality of the sensor's intrinsic magnetic coordinate system $\left(\alpha, \beta, \gamma\right.$ correspond to $v_{12} v_{23} v_{13}$ in [4]). The $\alpha$-angle is basically the error of orthogonality between $x$ - and $y$-axes, and $\beta$ and $\gamma$ def ne the error of $z$-axis orthogonality with respect to plane define by $x$ - and $y$ axes. Although the angle values $\alpha, \beta$, and $\gamma$ come from the mathematical processing of the scalar calibration algorithm, they are usually very stable in time and it has a sense to mention them with up to four decimal places precision. The $\alpha$-angle is inf uenced by any combination of the extra turns in $x$ - and $y$-axes, while the $z$-axis extra turns have almost no visible effect (Fig. 6). The change is up to $\pm 0.121^{\circ}$ from its nominal value of $-0.432^{\circ}$ for combination of extra turns in $x$ - and $y$ - axes, which applies for sensor (02). The $\alpha$-angle exhibits linear dependence on the nonhomogeneity created by extra turns in $x$-axis, and unfortunately, the dependency was measured only in three points: negative gradient, no extra gradient, and positive gradient (Fig. 4). Similar dependency was observed also for the nonhomogeneity in $y$-axis. The $\beta$-angle was practically insensitive to variation in $y$ -

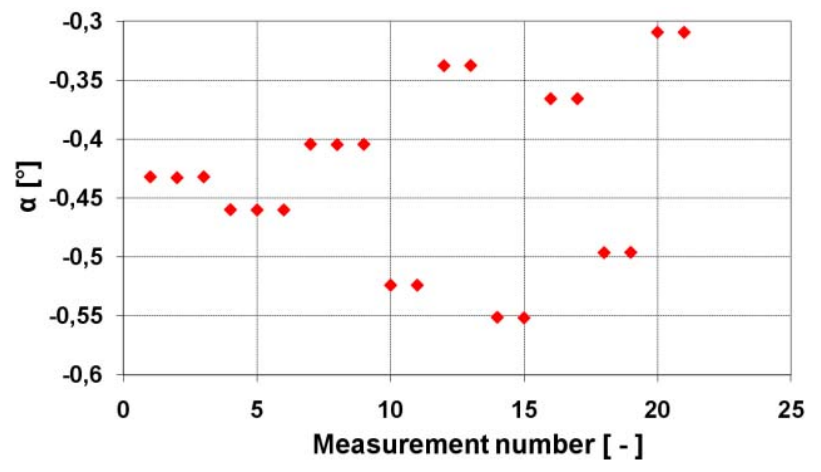

Fig. 6. $\alpha$-angle versus part of the measurements (no extra turns applied for numbers 1-3; numbers 4-6-turns added in $y$-axis; numbers 7-9-turns subtracted in $y$-axis; numbers 10 and 11-turns added in $x$-axis; numbers 12 and 13-turns subtracted in $x$-axis; numbers 14-21-various combinations of $x$ - and $y$-axis turns; and no change in $\alpha$ also for calibrations with only $z$-axis extra turns). Applies for the triaxial sensor (02).

\section{TABLE III}

EXTRA-TURns InfluENCE ON ORTHOGONALITY ANGLES (DoES Not INCLUde Their COMBINATIONS) For TRIAXIAL SENSOR (02)

\begin{tabular}{c|c|c|c} 
& a-angle & $\boldsymbol{\beta}$-angle & $\boldsymbol{\gamma}$-angle \\
\hline X-axis & $< \pm \mathbf{0 . 0 9 3 1 ^ { \circ }}$ & $< \pm \mathbf{0 . 0 1 3 1} 1^{\circ}$ & $< \pm 0.001^{\circ}$ \\
\hline Y-axis & $< \pm 0.0278^{\circ}$ & $< \pm 0.001^{\circ}$ & $< \pm 0.002^{\circ}$ \\
\hline Z-axis & $< \pm 0.002^{\circ}$ & $< \pm 0.002^{\circ}$ & $< \pm 0.116^{\circ}$
\end{tabular}

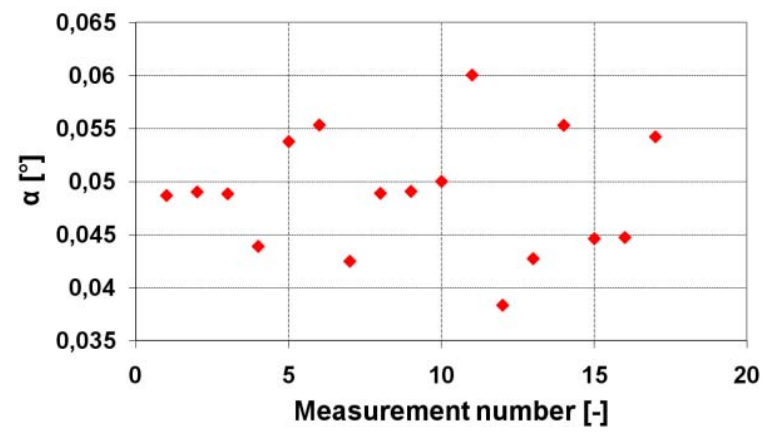

Fig. 7. $\alpha$-angle versus measurements (no extra turns applied for measurements numbers $1-3$; point numbers 4 and 5 are for nonhomogeneity applied in $x$-axis, numbers 6 and 7 in $y$-axis, and numbers 8 and 9 in $z$-axis; and numbers $10-17$ are for combinations of nonhomogeneity applied in two axes simultainously). Applies for triaxial sensor (04) with dual-ring-core inner topology.

and $z$-axes, but there was again a strong linear dependence for $x$-axis nonhomogeneity. There is almost no change for $\gamma$-angle for nonhomogeneity introduced in $x$ - and $y$-axes, and it stays within $\pm 0.002^{\circ}$ from its nominal value of $-0.0405^{\circ}$. But it is linearly sensitive to nonhomogeneity in $z$-axis. A summary of the inf uences is shown in Table III.

\section{B. Evaluation of the Field Homogeneity Influenc Versus Different Inner Topologies}

The results shown in Fig. 7 and Table IV conf rms our expectation that more symmetrical inner layout of triaxial sensor (04) should be less affected by the introduced nonhomogeneity. The measured change in orthogonality angles 
TABLE IV

EXTRA-TURNS INFLUENCE ON ORTHOGONALITY ANGLES (DOES NOT INCLUde Their COMBinATIONS) FOR TRIAXial SENSOR With DUAL-RING-CORE CONSTRUCTION (04)

\begin{tabular}{c|c|c|c} 
& $\boldsymbol{\alpha}$-angle & $\boldsymbol{\beta}$-angle & $\boldsymbol{\gamma}$-angle \\
\hline $\boldsymbol{X}$-axis & $< \pm 0.0049^{\circ}$ & $< \pm \mathbf{0 . 0 1 3 4 ^ { \circ }}$ & $< \pm 0.0001^{\circ}$ \\
\hline $\boldsymbol{Y}$-axis & $< \pm 0.0064^{\circ}$ & $< \pm 0.0003^{\circ}$ & $< \pm 0.0008^{\circ}$ \\
\hline $\boldsymbol{Z}$-axis & $< \pm 0.0001^{\circ}$ & $< \pm \mathbf{0 . 0 2} 2^{\circ}$ & $< \pm 0.0003^{\circ}$
\end{tabular}

TABLE V

Nonorthogonalities of the TRIAXIAL SENSORS. SENSOR (02) - Former Design, SENSors (03a) AND (03b) - With IMProved COMPENSATION Field HomOgeneity, AND SENSOR (04)-DUAL-RING-CORE INNER DESIGN

\begin{tabular}{c|c|c|c} 
Sensor & $\boldsymbol{a}$-angle & $\boldsymbol{\beta}$-angle & $\boldsymbol{\gamma}$-angle \\
\hline $\mathbf{( 0 2 )}$ & $-0.4387^{\circ}$ & $-0.1830^{\circ}$ & $-0.0476^{\circ}$ \\
\hline $\mathbf{( 0 3 a )}$ & $0.2973^{\circ}$ & $-0.0123^{\circ}$ & $0.1087^{\circ}$ \\
\hline $\mathbf{( 0 3 b )}$ & $0.4260^{\circ}$ & $-0.0469^{\circ}$ & $-0.0733^{\circ}$ \\
\hline $\mathbf{( 0 4 )}$ & $0.0468^{\circ}$ & $-0.2247^{\circ}$ & $-0.0270^{\circ}$
\end{tabular}

is practically ten times lower than in the case of sensor (02) listed in Table III.

Three new sensors with the improved compensation coil system feld homogeneity have been manufactured. Sensor (03a) uses three ring-core-based f uxgates, very similar in design to the previous generation of sensors that were used for the described measurements of f eld nonhomogeneity inf uence. Second sensor (03b) uses race-track core topology, which is supposed to provide lower noise. However, from scalar calibrations results, we did not fi d any significa $t$ improvement on the sensor parameters, mainly orthogonality error, although the results are not statistically relevant (two samples) (Table V). Failure to significa tly decrease the orthogonality error by improving the feld homogeneity led to the idea of using more symmetric inner topology, and one such is used in triaxial sensor (04).

The value of $\alpha$-angle for triaxial sensor (03a) is closer to its ideal value $\left(0^{\circ}\right)$, which could indicate the effect of improved feedback f eld homogeneity. On the other hand, the value is worse for triaxial sensor (03b) that uses race-track fuxgate sensors, which have magnetic cores with higher length than is the diameter of ring cores in (03a). Thus, the race-track sensor cores experience less homogenous compensation f eld.

The calibration results for triaxial sensor (04) indicate an Improvement, but it is again a statistically unreliable result (currently only one piece manufactured). The high value of $\beta$-angle might be caused in this case by error in the mechanical assembly as the 3-D-printed feedback f eld supports were not ideally precise.

\section{Evaluation of the Compensation Field Homogeneity Influenc on Offset-Temperature Dependence}

The offsets resulting from the scalar calibration were also affected by the introduced nonhomogeneity. For all three axes, temperature dependence has been observed. The temperature of the sensor has risen by $\sim 15{ }^{\circ} \mathrm{C}$ during the continuous scalar
TABLE VI

SENSOR OfFSETS VERSUS INTRODUCED NONHOMOGENEITY

\begin{tabular}{c|c|c|c} 
Sensor / Parameter & $\boldsymbol{X}$-axis & Y-axis & Z-axis \\
\hline 02- Absolute offset value & $-14.5 \mathrm{nT}$ & $125 \mathrm{nT}$ & $58 \mathrm{nT}$ \\
\hline 02- Rel. change for \pm Grad & $\pm 1.5 \mathrm{nT}$ & $\pm 10 \mathrm{nT}$ & $\pm 3 \mathrm{nT}$ \\
\hline 04- Absolute offset value & $116 \mathrm{nT}$ & $-263 \mathrm{nT}$ & $-364 \mathrm{nT}$ \\
\hline 04- Rel. change for \pm Grad & $\pm 2.3 \mathrm{nT}$ & $\pm 2.2 \mathrm{nT}$ & $\pm 7 \mathrm{nT}$
\end{tabular}
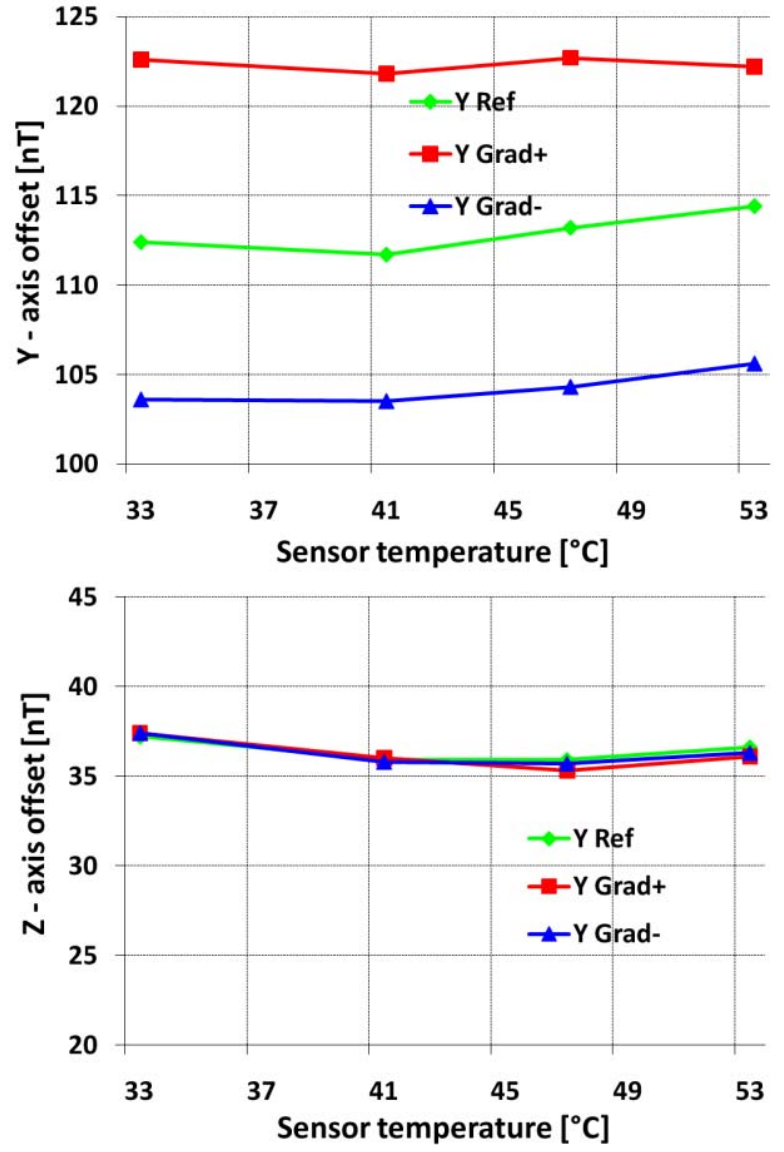

Fig. 8. Offset temperature dependence versus nonhomogeneity in $y$-axis $(\mathrm{Grad}+y$-axis extra turns in series, Grad- turns in antiseries, and Ref no extra turns).

calibration campaign by the energy dissipated in the excitation circuit and due to the rise of the ambient temperature. The temperature offset coeff cient is approximately $-0.3 \mathrm{nT} /{ }^{\circ} \mathrm{C}$.

After compensation on this effect, the offsets changed according to Table VI. However, the offsets are the least stable calibration parameter and the changes of $<2 \mathrm{nT}$ can be considered as noise. The same experiment was repeated also for the triaxial sensor (04) with a symmetrical inner design.

Another evaluation of offset temperature dependence was done in the magnetic shield. The results indicate that an absolute offset value is affected in the axis, where the nonhomogeneity is applied (Fig. 8). There was basically no visible change for the other two axes. The $y$-axis offset was thus inf uenced when there was a nonhomogeneity introduced in the $y$-axis feedback, there was no change on $x$ - or $z$-axis. We did not fnd any signif cant change in temperature dependence of the offset when introducing nonhomogeneity - it might be 
well below our measurement error. This was caused mainly by the measurement procedure: to switch between various extraturns conf guration the sensor was repeatedly removed from the magnetic shielding and then placed back. This resulted in uncertainty of the offset caused by remanence in the magnetic shield (below $5 \mathrm{nT}$ ) and also by temperature shocks applied to the sensor due to the procedure.

\section{CONCLUSION}

Several experiments and measurements were done to evaluate the inf uence of the feedback f eld homogeneity and the inner layout topology on a vectorially compensated triaxial sensor calibration parameters. An interesting feature observed is that the nonorthogonality angles were closer to their ideal zero values for nonideal feld homogeneity (in case of the nonsymmetrical three single-axis fuxgate design). With inseries turns applied (creating feedback feld nonhomogeneity in $x$-axis), the $\alpha$-angle value is closer to zero than for ideal no-extra turns condition. An explanation to this effect might be the sensor asymmetry in the coil system (Fig. 1) combined with the introduced nonhomogeneity. Nevertheless, from the results of calibration of the newly manufactured sensors (03a), (03b), and (04), we assume that the physical topology (inner layout) of the sensors is more important than the compensation feld homogeneity at this scale, since we did not fnd any dependence on offset temperature coeff cient with an artif cial nonhomogeneity. As long as each individual f uxgate sensor and appropriate feedback coil support material is stable enough, it is possible to use the results from scalar calibration and compensate for offset and even orthogonality temperature dependence.

\section{REFERENCES}

[1] G. Musmann, Fluxgate Magnetometers for Space Research. Norderstedt, Germany: Books on Demand, 2010

[2] F. Primdahl and P. A. Jensen, "Compact spherical coil for f uxgate magnetometer vector feedback," J. Phys. E, Sci. Instrum., vol. 15, no. 2, pp. 221-226, 1982.

[3] V. Petrucha and P. Kaspar, "Compact fluxgat sensor with a vector compensation of a measured magnetic f eld," in Proc. IEEE Sensors, Nov. 2010, pp. 1795-1798.

[4] J. M. G. Merayo, P. Brauer, F. Primdahl, J. R. Petersen, and O. V. Nielsen "Scalar calibration of vector magnetometers," Meas. Sci. Technol., vol. 11, no. 2, pp. 120-132, 2000.

[5] V. Petrucha, M. Janošek, and M. A. Azpúrua, "Fluxgate magnetometer vector feedback homogeneity and its influenc on sensor parameters," in Proc. IEEE Int. Instrum. Meas. Technol. Conf. (I2MTC), May 2014 pp. $460-463$.
[6] H. U. Auster et al., "The THEMIS f uxgate magnetometer," Space Sci. Rev., vol. 141, nos. 1-4, pp. 235-264, 2008.

[7] J. L. Kirschvink, "Uniform magnetic field and double-wrapped coil systems: Improved techniques for the design of bioelectromagnetic experiments," Bioelectromagnetics, vol. 13, no. 5, pp. 401-411, 1992.

[8] M. A. Azpurua, "A semi-analytical method for the design of coil-systems for homogeneous magnetostatic feld generation," Prog. Electromagn. Res. B, vol. 37, pp. 171-189, 2012.

[9] J. M. G. Merayo, "The vector fiel magnetometer (VFM) on swarm," in Proc. ESA's 2nd Swarm Int. Sci. Meeting, Potsdam, Germany, 2009.

[10] V. Petrucha and P. Kašpar, "Measurement of the temperature dependence of the sensitivity and orthogonality of a triaxial vector magnetometer," J. Elect. Eng., vol. 63. no. 7s, pp. 31-34, 2012.

[11] M. Janošek, J. Vyhnánek, A. Zikmund, P. Butvin, and B. Butvinová, "Effects of core dimensions and manufacturing procedure on fuxgate noise," Acta Phys. Polonica A, vol. 126, no. 1, pp. 104-105, 2014.

Vojtěch Petrucha was born in Přerov, Czech Republic, in 1982. He received the M.Sc. degree in measurements and instrumentation and the Ph.D. degree in calibration of magnetometers from the Faculty of Electrical Engineering, Czech Technical University in Prague, Prague, Czech Republic, in 2007 and 2012, respectively.

He is currently a Research Assistant with the Department of Measurement, Faculty of Electrical Engineering, Czech Technical University in Prague. His current research interests include the development and calibration of magnetic fiel sensors.

Michal Janošek was born in Varnsdorf, Czech Republic, in 1980. He received the M.Sc. degree in measurement and instrumentation from the Faculty of Electrical Engineering, Czech Technical University in Prague, Prague, Czech Republic, in 2007, where he is currently pursuing the Ph.D. degree.

He has co-authored over 20 journal papers. His current research interests include application of magnetic sensors in gradiometers and magnetometers.

Marco A. Azpúrua received the B.Sc. degree in telecommunications engineering from the Universidad Nacional Experimental Politécnica de la Fuerza Armada Nacional, Caracas, Venezuela, in 2008, and the M.Sc. degree in electrical engineering from the Universidad Central de Venezuela, Caracas, in 2013. He is currently pursuing the Ph.D. degree with the Universitat Politècnica de Catalunya, Barcelona, Spain.

He was a Researcher with the Applied Electromagnetics Laboratory, Department of Measurement Technology and Metrology, Electrical and Systems Engineering Center, Instituto de Ingeniería, Caracas. His current research interests include electromagnetic compatibility, antenna and microwave measurement technologies, and estimation of measurement uncertainty in complex systems and validation methods. 